\title{
Synthesis and Biological Activities of Jineol and Its Derivatives
}

\author{
Soon-Chang Cho, Md. Zakir Sultan, and Surk-Sik Moon ${ }^{*}$

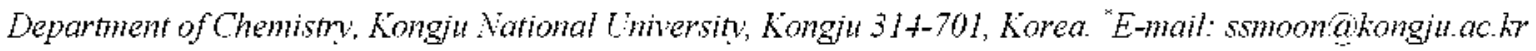 \\ Received Hav 11, 2008
}

Key Words : Jineol, Dilydroxyquinoline, Anticancer. Antimicrobial

Jineol (3,8-dihydroxyquinoline) (1). which exhibits cytotoxic and antifungal activities. has been isolated from the centipede Scolopendra subspinipes mutilans L. Koch. ${ }^{\prime}$ Recently. the species was used in the isolation of scolopendrine $(2)^{3}$ and 2.8-dilydroxy-3,4-dimethoxyquinoline (3) ${ }^{3}$ Compound 3 exhibits radical scavenging and low-density lipoprotein (LDL) antioxidant activities. ${ }^{3}$ Jineol and its dialkylated derivatives were also synthesized from 2-ntethosyaniline: however, their biological activities were not reported. Therefore, with regard to the biological activity of jineol derivatives. it might be interesting to introduce different functional groups at the two hydroxyl groups in compound 2. Here. we report a method of synthesizing jineol and its derivatives along with a view to obtain antibacterial, antifungal, and cy totoxic activities.<smiles>[R2]Oc1c([R])nc2c([R])c([R])ccc2c1[R]</smiles>

$1 \quad R^{1}=R^{2}=R^{3}=R^{4}=R^{5}=H$

$2 R^{1}=O H, R^{2}=M e, R^{3}=H$,

$R^{4}=4$-hydroxy-3-methoxybenzyl, $R^{5}=\mathrm{SO}_{3}$

$3 \mathrm{R}^{1}=\mathrm{OH}, \mathrm{R}^{2}=\mathrm{Me}, \mathrm{R}^{3}=$ OMe, $\mathrm{R}^{4}=\mathrm{R}^{5}=\mathrm{H}$

In this method, the reduction of 2-amino-3-methosybenzoic acid (4) with lithiun aluninum hydride followed by acetylation with acetic anhydride-triethylanine yjelded 2-acetanijo-3methosybenzyl acetate (5). Selective deacetylation with potassium carbonate in aqueous $\mathrm{MeOH}$ and subsequent oxidation with pyridium dicluromate in dichloromethane afforded a key intermediate. 2-acetamido-3-methoxybenzaldelyyde (7), which was unstable at room temperature Modified Friedlander condensation ${ }^{5}$ of aldeliyde 7 with benzylosyacetaldelyde and sodium hydroxide in ethanol afforded quinoline $8 \mathrm{in} 81 \%$ yield. Hydrogenation of compound 8 with hydrogen in the presence of $\mathrm{Pd} / \mathrm{C}$ or demethylation of the methosy group at C.8 of compound 8 with trimethylsilyl jodide in dry methylene chloride yielded jineol 8-metlyyl ether (9) or jineol 3-benzyl ether (10), respectively. Both demethylation of ether 9 with trimetlyy lsilyl iodide and debenzylation of ether $\mathbf{1 0}$ with hydrogen gas afforded jineol (1, 3.8-dilydrosy quinoline) in 30\% and quantitative yields, respectively. Spectroscopic data showed that the synthetic jineol was closely comparable to the natural product. ${ }^{1}$ 3-()Alkylated derivatives (11-20) of compound 1 were prepared from the reaction of 9 with various alkyl halides in basic dimethylsulfoxide in yields of $51-98 \%$, as shown in Scheme 1 .

The in vitro cytotoxicities of jineol and its derivatives were evaluated against human lung cancer cell A549 and mouse melanoma Bl6Fl cell lines. as shown in Table 1. Alkylation at $3-\mathrm{OH}$ and $8-\mathrm{OH}$ of compound 1 revealed no significant enhancement of activity as compared to the mother

Table 1. Antibacterial, antifungal, and cy totoxic activities of jineol and its derivatives ${ }^{2}$

\begin{tabular}{|c|c|c|c|c|c|c|c|c|c|c|}
\hline \multirow{2}{*}{$\begin{array}{l}\text { Compound } \\
\text { No }\end{array}$} & & & \multicolumn{4}{|c|}{ Bacterial strain ${ }^{b}$} & \multicolumn{2}{|c|}{ Fungal strain ${ }^{b}$} & \multicolumn{2}{|c|}{ Cancer cell line } \\
\hline & $R^{1}$ & $\mathrm{R}^{\hat{*}}$ & $\mathrm{EC}$. & SS & $\mathrm{BS}$ & $\mathrm{SA}$ & TM & $\mathrm{CA}$ & $\mathrm{Bl} 6 \mathrm{Fl}$ & A549 \\
\hline 1 & $\mathrm{H}$ & $\mathrm{H}$ & 64 & 16 & 32 & 32 & 64 & 64 & 30 & 2.2 \\
\hline 8 & $\mathrm{Me}$ & benzyl & 128 & - & 128 & - & - & - & 60 & 18 \\
\hline 9 & $\mathrm{Me}$ & $\mathrm{H}$ & - & - & - & - & - & - & - & - \\
\hline 10 & $\mathrm{H}$ & benzyl & - & 64 & 128 & 64 & 64 & 64 & 1.3 & 3.9 \\
\hline 11 & $\mathrm{Me}$ & 2-methylbenzyl & - & - & - & - & - & - & 9.6 & - \\
\hline 12 & $\mathrm{Me}$ & plnenethy1 & 128 & 16 & 16 & 16 & - & - & 6.5 & 16 \\
\hline 13 & $\mathrm{Me}$ & cyclohexy hnethyl & 128 & 16 & 16 & 16 & 64 & - & 3.8 & 9.5 \\
\hline 14 & $\mathrm{Me}$ & 2,3-dihydroxypropyl & - & - & - & - & - & - & - & - \\
\hline 15 & $\mathrm{Me}$ & 12-hydroxydodecyl & - & - & - & - & - & - & 3.0 & 11 \\
\hline 16 & $\mathrm{Me}$ & ethyl & nt & nt & nt & nt & nt & nit & - & - \\
\hline 17 & $\mathrm{Me}$ & isobutyl & nt & nt & nt & nt & nit & nit & 26 & 30 \\
\hline 19 & Me & hexadecy 1 & - & - & - & - & - & - & - & - \\
\hline 20 & $\mathrm{Me}$ & 2-ethylhesyl & - & 8 & 8 & 8 & - & - & 11 & 14 \\
\hline Control $^{:}$ & & & 1.0 & 0.5 & 0.5 & 0.5 & 4.0 & 4.0 & 7.5 & 10 \\
\hline
\end{tabular}

Data for antibiotic and cr to to sic activities are given in tems of minimum inhibition concentrations (MIC. $\mu \mathrm{g}$ mL) and $50^{\circ}$. inhibitory concentrations (IC 3 . prg $\mathrm{mL}$ ). respectively; "- "sign indicates no activit below $128 \mu \mathrm{g} / \mathrm{mL}$ for bacterial and fingal strains and 40 , th mL for cancer cell lines; "nt" indicates that

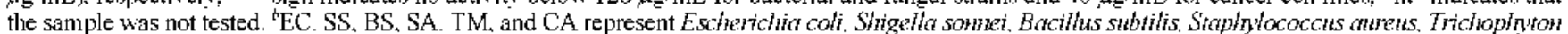
mentagroptytes, and Coftida abiconis, respectively. 'B $6 \mathrm{FI}$ and $\mathrm{A} 549$ cell lines originated from mouse melanoma and human lung cancer, respectively. "Tetracycline. ketoconacole, and cisplatin were used as positive controls for antibacterial. antifungal. and cytotoxicity: assay's. respectively: 


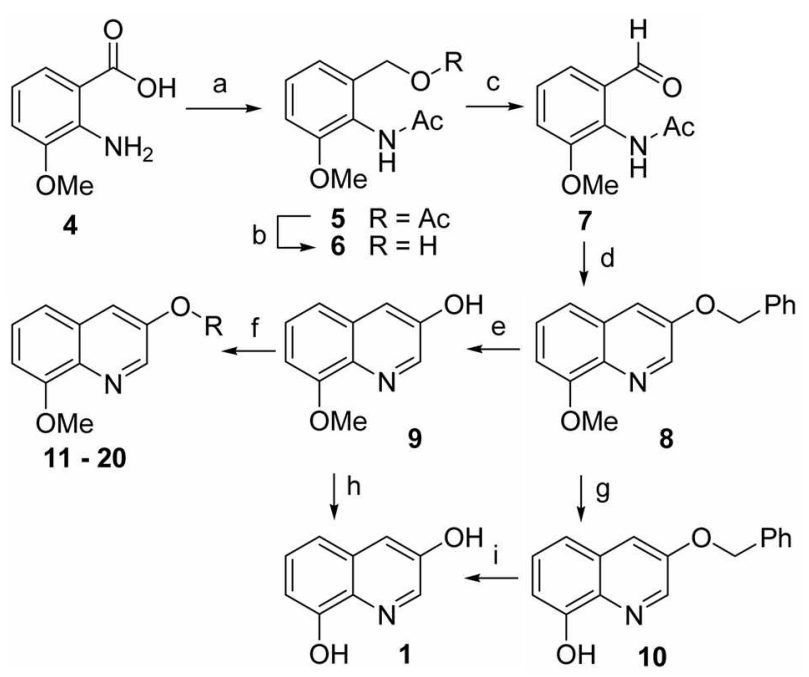

Scheme 1. Synthesis scheme of jineol and its derivatives. (a) $\mathrm{LAH}$, THF, reflus, 2 h: $\mathrm{Ac}_{2} \mathrm{O}, \mathrm{Et} \mathrm{t}_{3} \mathrm{~N}$, THF, rt, overnight. $71 \%$ : (b) $\mathrm{K}_{2} \mathrm{CO}_{3}$, $10 \%$ ac. $\mathrm{MeOH}$, rt, 20 min, $95 \%$, (c) $\mathrm{PDC}, \mathrm{CH}_{2} \mathrm{Cl}_{2}$, rt, oventight, $89 \%$, (d) benzvloxyethanal, $\mathrm{NaOH}$, EtOH reflux, $3 \mathrm{~h}, 81 \%$, (e) $\mathrm{H}_{2}$, $\mathrm{Pd} / \mathrm{C}, \mathrm{MeOH}$, it, quantitative: (f) alkyl halides, $\mathrm{K}_{2} \mathrm{CO}_{3}, \mathrm{DMSO}, 90$ ${ }^{\circ} \mathrm{C}$, overnight: (g) $\mathrm{Me}_{3} \mathrm{Si}-\mathrm{I}, \mathrm{CH}_{2} \mathrm{Cl}_{2}$, it, $24 \mathrm{~h}, 50 \%$; (h) Me $\mathrm{Me}_{3} \mathrm{Si}-\mathrm{I}$, $\mathrm{CH}_{2} \mathrm{Cl}_{2}, \mathrm{rt}, 24 \mathrm{~h}, 30 \%$; (i) $\mathrm{H}_{2}, \mathrm{Pd} / \mathrm{C}, \mathrm{MeOH}$, rt, quantitative

compound (1). Furthermore. the synthetic compounds were tested against gran positive bacterial strains (B. subtilis and $S$. atrens). gran negative bacterial strains ( $E$. coll and $S$. somnei). and fungal strains ( $T$. mentagrophytes and $C$. albicans). Compounds 12. 13. and 20 with phenethyl. cyclohexylnethyl. and 2-ethylhexyl groups at $3-\mathrm{OH}$, respectively. showed activity moderately higher or comparable to that of compound 1 against the bacterial strains (Table 1). On the other hand. other compounds like 15, 18, and 19, which bear long lipophilic alkyl groups showed no activity below 128 $\mu \mathrm{g} / \mathrm{mL}$ against all the strains tested.

\section{Experimental Section}

Melting points were measured using a Fisher melting point apparatus and the values are reported uncorrected. NMR spectra were recorded on a varian Mercury $400 \mathrm{MHz}$ spectroneter in deuterated methanol, acetone, or clloroform. Chemical shifts are expressed in ppm $(\delta)$ and referenced to methanol- $d_{4}\left(\delta_{\mathrm{H}} 3.31\right.$ and $\left.\delta_{-} 49.15\right)$. acetone- $d_{6}\left(\delta_{\mathrm{H}} 2.05\right.$ and $\delta_{-}$29.92). and $\mathrm{CDCl}_{3}\left(\delta_{\mathrm{H}} 7.27\right.$ and $\left.\delta_{-} 77.23\right)$ as internal standards. IR spectra were recorded on a Perkin Elnter BX FT-IR spectrometer with $\mathrm{KBr}$ pellets: the spectra are reported in wave numbers $\left(\mathrm{cm}^{-1}\right)$. Mass spectra were measured on a JEOL HXII0A Tanden HR mass spectrophotometer (electron impact mode) at the Korea Basic Science Institute. Routine monitoring of reactions was perfomed on a precoated silica gel plastic plate (Kieselgel $60 . \mathrm{F}_{2 \leq 4} .20 \times 20 \mathrm{~cm}$. $0.25 \mathrm{~nm}$ thick, Merck). Colunn chrontography was accomplished using silica gel (Merck. 60) with the eluent indicated. The starting synthetic materials were purchased from Aldrich Chemical Co. Tetrahydrofuran (THF) was distilled from sodium/potassium in argon atmosphere prior to use. Methylene chloride $\left(\mathrm{CH}_{2} \mathrm{Cl}_{2}\right)$ was distilled from calcium hydride.
All moisture-sensitive reactions were carried out in argon atmosphere using oven-dried glassware. The optical density for a 96-well microplate was measured on a Tecan Sunrise microplate reader (model A-5080) at $520 \mathrm{~mm}$. Brucella broth (BB) and potato dextrose broth (PDB) were purchased from Becton Dickinson and Company. All bacterial and fungal strains were purchased from the Korean Collection for Type Cultures (KCTC). Bacillus subilis (KCTC 1021). Siaphylococcus atreus (KCTC 1621). Eschenchia coli (KCTC 1039) and Shigella somei (KCTC 2009) were used for antibacterial assay. Candida albicans (KCTC 7121) and Trichophyton mentagrophytes (KCTC 6085) were used for antifungal assay. Human lung carcinoma A-549 and mouse melanoma Bl6-Fl cell lines were purchased from the Korean Cell Line Bank. Tetracycline, ketoconazole. and cisplatin were purchased from Signa Aldrich Co.

2-Acetamido-3-methoxybenzyl acetate (5). 2-Amino-3methoxybenzoic acid $4(6.0 \mathrm{~g}$. $36 \mathrm{mmol})$ was added in a small portion to a solution of lithium aluminum hydride (LAH) (2 g. $53 \mathrm{mmol})$ in dry THF $(50 \mathrm{~mL})$ in an ice bath for $10 \mathrm{~min}$ in argon atmosphere. The mixture was stirred for $2 \mathrm{~h}$ at room temperature. All additional amount of LAH (l g. 26 numol) was added and stirred for $2 \mathrm{~h}$ at room temperature followed by refluxing for $12 \mathrm{~h}$. The reaction mixture was diluted with methanol $(20 \mathrm{~mL})$ and sodium hydroxide solution ( $\mathrm{M}, 5 \mathrm{~mL}$ ), and it was then filtered using celite. The filtrate was concentrated and extracted with ethyl acetate $(50 \mathrm{~mL} \times 4)$. The combined organic layers were dried over anhydrous magnesium sulfate. Concentration afforded 2-amino-3-methoxybenzyl alcohol as brown oil [5.17 g: TLC (5:5 hexane ethyl acetate) $\left.R_{f} 0.41\right]$. which was subjected to acetylation without purification. The alcohol (5.17 g. $33.8 \mathrm{mmol}$ ) was added to a solution of acetic anhydride (8 $\mathrm{mL}, 78 \mathrm{mmol}$ ) and triethylamine (12 $\mathrm{mL} .118 \mathrm{mmol}$ ) in THF $(15 \mathrm{~mL})$ and stirred at room temperature for $6 \mathrm{~h}$. The reaction mixture was diluted with ethyl acetate $(400 \mathrm{~mL})$. washed with brine $(40 \mathrm{~mL} \times 2)$, and dried over anhydrous magnesium sulfate. Concentration afforded acetate $\mathbf{5}$ as a white solid. An authentic sample was obtained as a white cry stalline solid by trituration with $8: 2$ hexane-EtOAc $(6.4 \mathrm{~g} .71 \%$ yield from 4): $\mathrm{mp}: 98^{\circ} \mathrm{C}$ : IR $(\mathrm{KBr}): v_{\max } 3244.1748 .1656 .1542 .1483$. 1240. 1041, 770: ${ }^{1} \mathrm{H}$ NMR (acetone-d. $400 \mathrm{MHz}$ ): $\delta 7.23(\mathrm{lH}$. t. $J=8.0 \mathrm{~Hz}), 7.00(\mathrm{lH}, \mathrm{d} . J=8.0 \mathrm{~Hz}), 6.98(\mathrm{lH}, \mathrm{d} . J=8.0 \mathrm{~Hz})$. $5.03(2 \mathrm{H}, \mathrm{s}) .3 .81(3 \mathrm{H} . \mathrm{s}) .2 .10(3 \mathrm{H}, \mathrm{s}) .2 .05(3 \mathrm{H} . \mathrm{s}):{ }^{13} \mathrm{C}$ NMR (acetone- $d_{6}, 100 \mathrm{MHz}$ ): $\delta 170.6 .169 .1,154.9 .135 .7,127.6$, $125.3,120.1,111.2 .63 .3,56.1,23.0,20.8$; EIMS: $m / 2237$ $\left(\mathrm{M}^{-}, 45\right), 194(50), 176(21), 152(98), 134(55), 106(100)$.

2-Acetamido-3-methoxybenzyl alcohol (6). Acetate 5 $(6.4 \mathrm{~g} .27 \mathrm{mmol})$ was added to a solution of $\mathrm{K}_{2} \mathrm{CO}_{3}(1.0 \mathrm{~g}$. $7.2 \mathrm{mmol})$ in $\mathrm{MeOH}(25 \mathrm{~mL})$ and $\mathrm{H}_{2} \mathrm{O}(5 \mathrm{~mL})$. The mixture was stirred at room temperature for $20 \mathrm{~min}$ and diluted with saturated anmonium chloride solution $(150 \mathrm{~mL})$. The solution was extracted with ethyl acetate $(100 \mathrm{~mL} \times 10)$. The combined organic layers was washed with brine $(20 \mathrm{~mL} \times 2)$ and dried over anhydrous magnesium sulfate. Concentration and trituration with EtOAc-hexane afforded alcohol 6 as a white solid (4.97 g. $95 \%$ yield): mp: $133^{\circ} \mathrm{C}$ : IR (KBr): $v_{\text {max }}$ 
3213. 3041. 1654, 1542, 1484. 1283, 1044, 777: ${ }^{1} \mathrm{H}$ NMR (acetone- $d 6,400 \mathrm{MHz}): \delta 7.22(\mathrm{lH}, \mathrm{t}, J=7.8 \mathrm{~Hz}) .7 .11(\mathrm{lH}$. d. $J=7.6 \mathrm{~Hz}), 6.93(\mathrm{lH} . \mathrm{d}, J=8 \mathrm{~Hz}) .4 .45(2 \mathrm{H}, \mathrm{s}), 3.80(3 \mathrm{H}$. s). $2.14(3 \mathrm{H}, \mathrm{s}) ;{ }^{13} \mathrm{C} \mathrm{NMR}$ (acetone- $\left.d_{6}, 100 \mathrm{MHz}\right): \delta 170.3$. $154.7 .140 .8,127.7,124.6,121.2,110.5,61.6 .56 .0,23.1$.

2-Acetamido-3-methoxy-benzaldehyde (7). Alcohol 6 (5.0 g. $26 \mathrm{mumol})$ and pyridium dicluromate ( $15 \mathrm{~g} .45 \mathrm{~nm}$ ol) were dissolved in dry methylene chloride $(100 \mathrm{~mL})$. The mixture was stirred for $12 \mathrm{~h}$ at room temperature. The reaction mixture was passed through a short colunu of silica gel with the elution of ethyl acetate to obtain 2-acetamido-3methoxy-benzaldehyde 7 as a colorless crystalline solid (4.4 g. $89 \%$ yield). The solid was subjected to subsequent reaction without further purification; IR $(\mathrm{KBr})$ : $v_{\max } 3241$. 1697. 1664. 1590, 1528, 1459. 1271, 1074, 771: ${ }^{1} \mathrm{H}$ NMR (acetone-d. $400 \mathrm{MHz}$ ): $\delta 9.92(\mathrm{lH} . \mathrm{s}), 7.40-7.38$ (IH. m), 7.32 $7.30(2 \mathrm{H} . \mathrm{ml}) .3 .89(3 \mathrm{H}, \mathrm{s}) .2 .2 \mathrm{l}(3 \mathrm{H}, \mathrm{s}) ;{ }^{13} \mathrm{C}$ NMR (acetone$\left.d_{6}, 100 \mathrm{MHz}\right): \delta 189.6,170.6 .153 .6 .132 .2,129.1 .127 .0,119.0$, 116.5. 56.5, 23.2: TLC (1:9 hexane ethyl acetate): $R_{f} 0.3$.

3-Benzyloxy-8-methoxy quinoline (8). The aldehyde 7 (1.5 g. $7.8 \mathrm{mmol}$ ) and sodium hydroxide ( $1.8 \mathrm{~g} .45 \mathrm{mmol})$ were dissolved in ethanol. Benzyloxyethanal (1.8 miL. 12 mmol) was added dropwise to the aldehyde solution for 15 min at room temperature. The reaction mixture was stirred at $50{ }^{\circ} \mathrm{C}$ for 30 min and an additional amount of benzyloxyacetaldelyyde ( $1 \mathrm{~mL} .6 .7 \mathrm{~mm}$ ol) was added and stired for $3 \mathrm{~h}$ at the same temperature. The reaction misture was diluted with EtOAc $(500 \mathrm{~mL})$. washed with brine $(100 \mathrm{~mL})$, and dried over anhydrous magnesium sulfate. Filtration and concentration yielded a brown oil, which was purified by silica gel column chromatography (30 id $\times 250 \mathrm{nmm}$. $70-230$ mesh) to obtain quinoline ether $\mathbf{8}$ as a brown cry stalline solid (1.7 g. 81\% yield); mp: $74^{\circ} \mathrm{C}$; ${ }^{1} \mathrm{H}$ NMR (acetone- $d 6.400 \mathrm{MHz}$ ): $\delta 8.63(\mathrm{lH} . \mathrm{d} . J=2.8 \mathrm{~Hz}), 7.71(\mathrm{lH}, \mathrm{d}, J=2.8 \mathrm{~Hz}), 7.56-7.55$ $(2 \mathrm{H}, \mathrm{m}), 7.46-7.4 \mathrm{l}(3 \mathrm{H}, \mathrm{m}), 7.38-7.34(2 \mathrm{H}, \mathrm{m}), 7.00(\mathrm{lH}, \mathrm{dd}$. $J=7.6 .1 .2 \mathrm{~Hz}), 5.28(2 \mathrm{H}, \mathrm{s}), 3.98(3 \mathrm{H}, \mathrm{s}) ;{ }^{13} \mathrm{C}$ NMR (acetone$\left.d_{6}, 100 \mathrm{MHz}\right) ; \delta 156.8 .153 .5 .143 .1,137.5,136.2 .131 .1$. $129.3(2 \mathrm{C}) .128 .8 .128 .6(2 \mathrm{C}) .128 .3 .119 .5 .114 .3 .106 .7$. 70.7. 56.0. EIMS: $m=265\left(\mathrm{M}^{-} .25\right), 116(9), 91(100)$.

3-Hydroxy-8-methoxyquinoline (9). Quinoline ether 8 (570 $\mathrm{mg} .2 .2 \mathrm{mmol}$ ) was dissolved in methanol $(15 \mathrm{~mL})$ and $\mathrm{Pd} / \mathrm{C}(50 \mathrm{mg})$ was added. The mixture was flushed with hỵdrogen gas and stirred overnight in an atmosphere of hydrogen at room temperature. The reaction mixture was concentrated and diluted with EtOAc. It was then passed through a short column of celite with EtOAc elution. Concentration afforded alcohol 9 as a yellow solid in quantitative yield: mp: $198^{\circ} \mathrm{C}:{ }^{1} \mathrm{H}$ NMR (acetone- $d_{6}, 400 \mathrm{MHz}$ ): $\delta 8.59$ (iH. d. $J=2.8 \mathrm{~Hz}), 7.52(\mathrm{lH} . \mathrm{d}, J=2.8 \mathrm{~Hz}) .7 .40(1 \mathrm{H}, \mathrm{t}, J=8.0 \mathrm{~Hz}$. H-6). $7.28(1 \mathrm{H} . \mathrm{dd} J=8.4 .1 .2 \mathrm{~Hz}) .6 .95(1 \mathrm{H} . \mathrm{d} . J=7.6 .0 .8$ Hz) $3.96(3 \mathrm{H}, \mathrm{s} . \mathrm{OMe}):{ }^{13} \mathrm{C}$ NMR (acetone-d, $\left.100 \mathrm{MHz}\right): \delta$ 156.6. 152.2. 142.4, 135.7. 131.6. 128.0. 128.6, 119.0, 116.4. 105.9. 55.9: EIMS: $m / 2175\left(\mathrm{M}^{+}, 89\right)$. $146(100), 116(21)$

3-Benzyloxy-8-hydroxyquinoline (10). Trumethylsilyl iodide $(0.4 \mathrm{~mL}, 1.65 \mathrm{mmol})$ was added dropwise to a solution of quinoline ether $8(200 \mathrm{mg} .0 .75 \mathrm{mmol})$ in dry $\mathrm{CH}_{2} \mathrm{Cl}_{2}(8 \mathrm{~mL})$ in argon atmosphere and the mixture was stirred at room temperature for $24 \mathrm{~h}$. The mixture was then diluted with EtOAc $(20 \mathrm{~mL})$ and a saturated $\mathrm{NaHCO}_{3}$ solution $(20 \mathrm{~mL})$ was added. The organic layer was washed with brine $(10 \mathrm{~mL}$ $\times 2$ ) and dried over anhydrous $\mathrm{MgSO}_{4}$. After filtration and concentration, the residue was purified by silica gel column chromatography (30 id $\times 250 \mathrm{~mm}, 70-230$ mesh. $4: 6$ hexane: EtOAc) to obtain benzyl ether 10 as a brown solid $(94.7 \mathrm{mg}$. $50 \%$ yield): $m p: 104{ }^{\circ} \mathrm{C} ;{ }^{1} \mathrm{H}$ NMR $\left(\mathrm{CD}_{3} \mathrm{OD}, 400 \mathrm{MHz}\right): \delta$ $8.56(\mathrm{lH}$. d. $J=2.8 \mathrm{~Hz}) .7 .66(\mathrm{lH}$. d. $J=2.8 \mathrm{~Hz}) .7 .50(2 \mathrm{H}$, d. $J=7.2 \mathrm{~Hz}), 7.33-7.42(4 \mathrm{H}, \mathrm{m}), 7.25(\mathrm{lH} . \mathrm{d}, 8.0) .6 .92$ (lH. d, $J=7.2 \mathrm{~Hz}), 5.23$ (2H. s); ${ }^{13} \mathrm{C}$ NMR (CD 3 OD. 100 $\mathrm{MHz}): \delta 154.39 .154,35,143.2,137.9,135.2 .131 .4,129.6$ (2C), 129.3, 129.2, 128.8(2C), 118.4, 115.4, 109.9, 71.4.

\section{Jineol (3,8-dihydroxyquinoline) (1)}

Method (a): Trimethylsilyl iodide $(0.2 \mathrm{~mL}, 0.83 \mathrm{mmol})$ was added dropwise to a solution of methyl ether $9(80 \mathrm{mg}$. $0.46 \mathrm{mmol})$ in dry $\mathrm{CH}_{2} \mathrm{Cl}_{2}(8 \mathrm{~mL})$ in argon atmosphere and the mixture was stirred at room temperature for $24 \mathrm{~h}$. The misture was then diluted with EtOAc $(15 \mathrm{~mL})$ and a saturated $\mathrm{NaHCO}_{3}$ solution $(15 \mathrm{~mL})$ was added. The organic layer was washed with brine $(5 \mathrm{~mL} \times 2)$ and dried over anthydrous $\mathrm{MgSO}_{4}$. After filtration and concentration. the residue was purified by silica gel column chromatography (30 id $\times 250$ num, 70-230 mesh, 1:9 hexane:EtOAc) to obtain jineol 1 as a brown solid ( $22 \mathrm{mg} .30 \%$ y ield)

Method (b): Benzyl ether 10 (95 $\mathrm{mg} .0 .38 \mathrm{mmol})$ was dissolved in $\mathrm{MeOH}$ and $\mathrm{Pd} / \mathrm{C}(5 \mathrm{mg}$ ) was added. The mixture was flushed with hydrogen gas and stirred overnight in hydrogen atmosphere at room temperature. The reaction mixture was then passed through a short column of celite with EtOAc elution and concentrated to obtain alcohol 1 as a brown solid in quantitative yield: this solid was identical to the sample obtained by method (a).

General procedure for preparing 3-alkyloxy-8-methoxyquinolines (11-20). Compound 9 (50 $\mathrm{mg}, 0.28 \mathrm{mmol}$ ) was dissolved in DMSO (3 mL) and $\mathrm{K}_{2} \mathrm{CO}_{3}(38 \mathrm{mg}, 0.28$ mmol) was added. The mixture was stirred for $10 \mathrm{~min}$. The following alkyl halides (1.5 eq. each) were then added: 2methỵlbenzyl bromide: phenethyl bromide: cyclohexỵlmethỵl bromide, 2,3-dihỵdroxypropyl bromide: 12-hydroxyddodeçyl bromide: ethyl bromide. isobutyl bromide: octadecyl bromide: hexadecyl bromide: and 2-ethylhexyl bromide for compounds 11 to 20 , respectively. The mixture was stirred overnight at $80^{\circ} \mathrm{C}$. The reaction mixture was diluted with EtOAc (100 $\mathrm{mL})$. washed with brine $(100 \mathrm{~mL})$, and dried over anhydrous magnesium sulfate. Filtration and concentration followed by silica gel columu chromatography ( $30 \mathrm{id} \times 250 \mathrm{~nm}, 70-230$ mesh) afforded the corresponding ethers 11-20 in specified yields for each compound.

3-(2-Methyl)benzyloxy-8-methoxy quinoline (11): A brown solid (51.8 mg. 65\%): mp: $119^{\circ} \mathrm{C}:{ }^{1} \mathrm{H} \mathrm{NMR}\left(\mathrm{CDCl}_{3}\right.$. $400 \mathrm{MHz}$ ): $\delta 8.72$ (lH. d. $J=3.2 \mathrm{~Hz}$ ), $7.82(\mathrm{lH} . \mathrm{d} . J=3.2 \mathrm{~Hz}$ ). $7.70(\mathrm{lH}, \mathrm{d}, J=8.4 \mathrm{~Hz}), 7.47(\mathrm{lH}$, brd,$J=7.2 \mathrm{~Hz}) .7 .24-7.30$ $(4 \mathrm{H}, \mathrm{m}), 6.81(\mathrm{lH}, \mathrm{d} J=8.4 \mathrm{~Hz}) .5 .23(2 \mathrm{H}, \mathrm{s}) .4 .07(3 \mathrm{H}, \mathrm{s})$. $2.44(3 \mathrm{H}, \mathrm{s}):{ }^{13} \mathrm{C}$ NMR $\left(\mathrm{CDCl}_{3}, 100 \mathrm{MHz}\right) ; \delta 155.6,154.1$. 143.9. 137.3. 135.9. 133.7, 131.0, 130.9, 129.45, 129.41. 129.1. $1264.113 .7,111.0,106.3,69.3,56.3,19.2$. 
3-Phenethyloxy-8-methoxy quinoline (12): A light brown solid (48.6 mg. $61 \%$ ). mp: $79^{\circ} \mathrm{C}:{ }^{1} \mathrm{H} \mathrm{NMR}\left(\mathrm{CDCl}_{3} .400 \mathrm{MHz}\right)$ : $\delta 8.66(\mathrm{lH}, \mathrm{d} . J=2.8 \mathrm{~Hz}) .7 .67(1 \mathrm{H}, \mathrm{d} . J=8.4 \mathrm{~Hz}) .7 .66(1 \mathrm{H} . \mathrm{d}$. $J=2.8 \mathrm{~Hz}) .7 .26-7.36(6 \mathrm{H} . \mathrm{ml}) .6 .79(\mathrm{lH}, \mathrm{d} . J=8.4 \mathrm{~Hz}) .4 .36$ $(2 \mathrm{H} . \mathrm{t} . J=7.2 \mathrm{~Hz}) .4 .06(3 \mathrm{H} . \mathrm{s}) .3 .2 \mathrm{l}(2 \mathrm{H} . \mathrm{t} . J=7.2 \mathrm{~Hz}) \cdot{ }^{13} \mathrm{C}$ NMR $\left(\mathrm{CDCl}_{3} .100 \mathrm{MHz}\right): \delta 155.6,154.1,143.9,137.8,135.8$. $131.0,129.4,129.3(2 \mathrm{C}), 128.9(2 \mathrm{C}), 127.0,113.3,111.0$. $106.2 .69 .4,56.3,35.6$.

3-Cyclohexylmethyloxy-8-methoxy quinoline (13): A pale brown solid (75.9 mg. $98 \%$ ): mp: $90{ }^{\circ} \mathrm{C}:{ }^{1} \mathrm{H} \mathrm{NMR}\left(\mathrm{CDCl}_{\hat{3}}\right.$. $400 \mathrm{MHz}): \delta 8.65(\mathrm{lH}, \mathrm{d} . J=2.8 \mathrm{~Hz}), 7.40(1 \mathrm{H}, \mathrm{t} . J=8.0 \mathrm{~Hz})$, $7.31(1 \mathrm{H}$ d. $J=2.8 \mathrm{~Hz}) .7 .26(1 \mathrm{H} . \mathrm{d} . J=8.0 \mathrm{~Hz}) .6 .88(1 \mathrm{H} . \mathrm{d} . J$ $=8.0 \mathrm{~Hz}), 4.06(3 \mathrm{H}, \mathrm{s}) .3 .86(2 \mathrm{H} . \mathrm{d} . J=6.0 \mathrm{~Hz}), 1.69-1.92(6 \mathrm{H}$, m). $1.05-1.37(5 \mathrm{H} . \mathrm{m}):{ }^{13} \mathrm{C} \mathrm{NMR}\left(\mathrm{CDCl}_{3} .100 \mathrm{MHz}\right): \delta 155.8$. 153.5. 143.5. 135.2. 130.5. 127.6. 118.8. 113.2. 105.3. 73.9. $56.1 .37 .8,30.1,26.6,26.0$.

3-(2,3-Dihydroxypropyl)oxy-8-methoxy quinoline (14): A pale brown solid ( $36.3 \mathrm{mg} .51 \%$ ); mp: $181{ }^{\circ} \mathrm{C},{ }^{1} \mathrm{H}$ NMR $\left(\mathrm{CD}_{3} \mathrm{OD} .400 \mathrm{MHz}\right.$ ): $\delta 8.55$ (1H. d $J=3.2 \mathrm{~Hz}$ ). 7.69 (1H. d. $J=$ $3.2 \mathrm{~Hz}$ ). 7.47 (lH. d. $J=7.6 \mathrm{~Hz}$ ). 7.39 (lH. d. $J=7.6 \mathrm{~Hz}$ ). 7.04 $(\mathrm{IH} . \mathrm{d}, J=7.6 \mathrm{~Hz}) \cdot 4.24(\mathrm{lH}$. dd. $J=9.6 .4 .4 \mathrm{~Hz}), 4.15(\mathrm{IH} . J=$ $9.6 .6 .0 \mathrm{~Hz}) .4 .06(1 \mathrm{H} . \mathrm{m}) .4 .03(3 \mathrm{H} . \mathrm{s}) .3 .74(1 \mathrm{H} . \mathrm{dd} J=11.2$. $5.6 \mathrm{~Hz}), 3.71(1 \mathrm{H}$, dd. $J=11.2,5.6 \mathrm{~Hz}):{ }^{13} \mathrm{C}$ NMR $\left(\mathrm{CD}_{3} \mathrm{OD}\right.$, $100 \mathrm{MHz}): \delta 156.3,154.6,143.5,135.7,131.9,129.0 .120 .0$. 115.4. 107.0.71.5. 70.9.64.0.56.3.

3-(12-Hydroxydodecyl)oxy-8-methoxy quinoline (15): A white solid (102.7 mg. 91\%): $\mathrm{mp}: 52^{\circ} \mathrm{C}:{ }^{1} \mathrm{H} \mathrm{NMR}\left(\mathrm{CDCl}_{3}\right.$. $400 \mathrm{MHz}): \delta 8.66(1 \mathrm{H} . \mathrm{d} . J=3.2 \mathrm{~Hz}) .7 .41(1 \mathrm{H}, \mathrm{t}, J=8.0 \mathrm{~Hz})$. $7.34(1 \mathrm{H} . \mathrm{d} . J=3.2 \mathrm{~Hz}) .7 .27(1 \mathrm{H} . \mathrm{dd} J=8.0 .1 .2 \mathrm{~Hz}) .6 .89$ (1H. dd $J=8.0,1.2 \mathrm{~Hz}) .4 .07(2 \mathrm{H}, \mathrm{t}, J=6.8 \mathrm{~Hz}) .4 .06(3 \mathrm{H}, \mathrm{s})$, $3.63(2 \mathrm{H} . \mathrm{t} . J=6.8 \mathrm{~Hz}) .1 .86(2 \mathrm{H}, \mathrm{m}) .1 .56(2 \mathrm{H} . \mathrm{m}) .1 .50(2 \mathrm{H}$. m), 1.24-1.36 (1-4. m): ${ }^{13} \mathrm{C} \mathrm{NMR}\left(\mathrm{CDCl}_{3}, 100 \mathrm{MHz}\right): \delta 155.8$, $153.4 .143 .4 .135 .2,130.5,127.6 .118 .8$. 113.3. 105.4. 68.6.6.3. 56.1 .33 .0 .29 .80 .29 .75 (3C) $29.63 .29 .56 .29 .3,26.2 .25 .9$.

3-Ethoxy-8-methoxy quinoline (16): A brown oil (34.8 mg. $60 \%$ ): ${ }^{1} \mathrm{H} \mathrm{NMR}\left(\mathrm{CDCl}_{\hat{\jmath} .} 400 \mathrm{MHz}\right): \delta 8.69$ (1H. d. $J=$ $2.8 \mathrm{~Hz}) .7 .43(\mathrm{lH} . \mathrm{t}, J=8.0 \mathrm{~Hz}) .7 .38(\mathrm{lH} . \mathrm{d}, J=2.8 \mathrm{~Hz})$. $7.29(1 \mathrm{H} . \mathrm{dd} J=8.0 .1 .2 \mathrm{~Hz}), 6.91(1 \mathrm{H} . \mathrm{dd} . J=8.0 .1 .2 \mathrm{~Hz})$. $4.17(2 \mathrm{H}$ quartet $J=7.2 \mathrm{~Hz}) .4 .07(3 \mathrm{H} . \mathrm{s}) .1 .51$ (3H. t. $J=$ $7.2 \mathrm{~Hz}):{ }^{13} \mathrm{C}$ NMR $\left(\mathrm{CDCl}_{3}, 100 \mathrm{MHz}\right): \delta 155.5,153.2,143.1$, 135.0. 130.1 127.8. 118.8. 113.9. 105.7. 64.2, 56.2. 14.8.

3-Isobutoxy-8-methoxy quinoline (17): A pale brown solid (62.7 mg. 95\%): ${ }^{1} \mathrm{H} \mathrm{NMR}$ (CDCl $3.400 \mathrm{MHz}$ ): $\delta 8.67(\mathrm{lH}$. d. $J=3.0 \mathrm{~Hz}) .7 .40(1 \mathrm{H} . \mathrm{t} . J=8.0 \mathrm{~Hz}), 7.33(\mathrm{lH} . \mathrm{d}, J=3.0 \mathrm{~Hz})$, 7.27 (lH. dd. $J=8.0,1.2 \mathrm{~Hz}), 6.89(\mathrm{lH}, \mathrm{dd}, J=8.0,1.2 \mathrm{~Hz})$, $4.06(3 \mathrm{H} . \mathrm{s}) .3 .84(2 \mathrm{H} . \mathrm{d} J=6.0 \mathrm{~Hz}) .2 .17(1 \mathrm{H}$. septet. $J=6.8$ Hz). $1.07(6 \mathrm{H}, \mathrm{d}, J=6.8 \mathrm{~Hz}) ;{ }^{13} \mathrm{C} \mathrm{NMR}\left(\mathrm{CDCl}_{3}, 100 \mathrm{MHz}\right): \delta$ $155.8 .153 .5,143.5,135.2 .130 .5,127.6,118.8,113.3,105.4$. $74.9 .56 .1,28.4,19.5(2 \mathrm{C})$.

3-0ctadecyloxy-8-methoxy quinoline (18): A pale brown solid (88 mg. $74 \%$ ): mp: $57^{\circ} \mathrm{C}:{ }^{l} \mathrm{H}$ NMR $\left(\mathrm{CDCl}_{3} .400\right.$ MHz): $\delta 8.71(1 \mathrm{H}, \mathrm{s}) .7 .43(1 \mathrm{H}, \mathrm{t}, J=8.0 \mathrm{~Hz}) .7 .40(\mathrm{lH} . \mathrm{d}, J$ $=2.8 \mathrm{~Hz}) .7 .30(1 \mathrm{H} . \mathrm{d} J=8.4 \mathrm{~Hz}) .6 .93(1 \mathrm{H} . \mathrm{d} . J=8.0 \mathrm{~Hz})$. $4.09(2 \mathrm{H}, \mathrm{t} . J=6.8 \mathrm{~Hz}), 4.08(3 \mathrm{H}, \mathrm{s}) .1 .86(2 \mathrm{H} . \mathrm{m}) .1 .50(2 \mathrm{H}$. m) $1.25-1.37(28 \mathrm{H} . \mathrm{m}), 0.87(3 \mathrm{H}, \mathrm{t}, J=6.8 \mathrm{~Hz}){ }^{13} \mathrm{C}$ NMR $\left(\mathrm{CDCl}_{3}, 100 \mathrm{MHz}\right): \delta 155.3,153.5,143.1 .135 .0,130.6$. 128.0. 118.7. 113.9, $105.9,68.8,56.2 .32 .2,29.92(5 \mathrm{C})$.
29.88 (3C), 29.82. 29.78. 29.6 (2C). 29.3, 26.2. 22.9, 14.3.

3-Hexadecyloxy-8-methoxy quinoline (19): A pale brown solid (73 mg. $67 \%$ ): mp: $51{ }^{\circ} \mathrm{C}:{ }^{~} \mathrm{H} \mathrm{NMR}\left(\mathrm{CDCl}_{2} .400 \mathrm{MHz}\right.$ ): $\delta$ $8.78(\mathrm{lH} . \mathrm{s}) .7 .48(\mathrm{lH}, \mathrm{t} . J=8.0 \mathrm{~Hz}), 7.34(\mathrm{lH} . \mathrm{d} . J=2.8 \mathrm{~Hz}$ ), $7.31(1 \mathrm{H}, \mathrm{dd} . J=8.0 \mathrm{~Hz}), 6.97(1 \mathrm{H}, \mathrm{d} . J=6.8 \mathrm{~Hz}) .4 .11(2 \mathrm{H}, \mathrm{t} . J$ $=6.8 \mathrm{~Hz}), 4.10(3 \mathrm{H}, \mathrm{s}), 1.87(2 \mathrm{H} . \mathrm{m}) .1 .50(2 \mathrm{H}, \mathrm{m}), 1.25-1.34$ $(24 \mathrm{H}, \mathrm{m}) .0 .87(3 \mathrm{H}$. t. $J=6.4 \mathrm{~Hz}):{ }^{13} \mathrm{C}$ NMR $\left(\mathrm{CDCl}_{3}, 100\right.$ MHz): $\delta 155.3,153.5 .142 .2,135.2,130.6 .128 .0,118.7 .113 .9$. 105.9. 68.8, 56.3. 32.2. 29.9 (4C). 29.88 (2C), 29.8. 29.77, 29.6 (2C). 29.2. 26.2. 22.9, 14.3.

3-(2-Ethyl)hexyloxy-8-methoxy quinoline (20): A pale brown oil (61.5 mg. $75 \%$ ): ${ }^{1} \mathrm{H} \mathrm{NMR}$ ( $\mathrm{CDCl}_{3 .} 400 \mathrm{MHz}$ ): $\delta 8.66$ $(\mathrm{lH} . \mathrm{d}, J=2.8 \mathrm{~Hz}), 7.4 \mathrm{l}(\mathrm{lH}, \mathrm{t} . J=8.0 \mathrm{~Hz}) .7 .35(\mathrm{lH} . \mathrm{d} J=2.8 \mathrm{~Hz})$, $7.27(1 \mathrm{H}$ brd. $J=8.0 \mathrm{~Hz}) .689(1 \mathrm{H}$ brd. $J=8.0 \mathrm{~Hz}) .4 .06(3 \mathrm{H} . \mathrm{s})$.

Antibacterial and antifungal activity. The antimicrobial activities of the synthetic compounds were determined by the broth dilution method. ${ }^{6} \mathrm{~A}$ solution of the synthetic compounds in methanol was added in 24-ivell plates containing $1 \mathrm{~mL}$ of culture broth $(2.8 \mathrm{~g} \mathrm{BB}$ in $100 \mathrm{~mL}$ water for bacteria and $2.4 \mathrm{~g} \mathrm{PDB}$ in $100 \mathrm{~mL}$ water for fungi) for each microorganism and the wells were then two-fold serially diluted with culture broth. Overnight-precultured bacterial cells $\left(10^{\circ}\right.$ $\mathrm{CFU} / \mathrm{mL}$ ) were inoculated in the wells and incubated at 37 ${ }^{\circ} \mathrm{C}$ for bacteria and $26^{\circ} \mathrm{C}$ for fungi for $48 \mathrm{~h}$. The lowest concentration that prevented the visible growth of microorganisms was reported as the minimum inhibitory concentration (MIC).

Cytotoxicity assay. Cytotoxicity of the cancer cell lines was measured by a colorimetric sulforhodamine B (SRB) assay. Exponentially growing cells were harvested and suspended in the culture media ( $100 \mu \mathrm{L}$, RPMI-1640) in a 96-well plate (seeding density for A-549 and B16Fl: $1 \times 10^{5}$ and $2 \times 10^{+}$cells $/ \mathrm{mL}$. respectively). After $24 \mathrm{~h}$ of incubation at $37^{\circ} \mathrm{C}$ in humidified $5 \% \mathrm{CO}_{2}$, serially diluted test solutions (100 $\mu \mathrm{L}$ in RPMI media) were added to the wells and incubated further for $48 \mathrm{~h}$. The cells were fixed with $50 \%$ trichloroacetic acid and stained for $30 \mathrm{~min}$ with an SRB solution. The unbound dye was removed by $1 \%$ acetic acid (four times) and the protein-bound dye was extracted with $10 \mathrm{mM}$ tris base ( $\mathrm{pH} \mathrm{10.5)} \mathrm{for} 5 \mathrm{~min}$. The optical density of the released dye was measured at $520 \mathrm{~nm}$ using a microplate reader. The results were expressed as the concentration at which there was $50 \%$ inhibition $\left(I C_{50}\right)$.

Acknowledgments. This work was supported by a grant from Kongju National University.

\section{References}

1. Moon. S.-S.: Cho. N.: Shin, J:- Seo, Y.: Lee, C. O.: Choi. S. U. $d$ Sat. Prod 1996, 59,777.

2. Noda. N.: Yashiki. Y: Nakatani. T.: Myyahara. K.: Du. X. M. Chem. Pharm. Bull. 2001. 19.930.

3. Yoon. M.-A.: Jeong. T.-S.: Park. D.-S.: Xu. M.Z.: Oh. H.-W: Song. K.-B.: Lee. W. S.: Park. H.-Y. Biol. Phom. Bull 2006. 29.735.

4. Yoshinobu. T.: Hiroyuki. Y.; Manami, N.: Yoshinobu, G. Heterocycles 1998. +8. 2379.

5. Boger. D. L.: Chen. J. H. J. Org. Chem. 1995. 60. 7369.

6. Sultan, M. Z.; Jeon, Y. M.: Moon, S.-S. Planta Med 2008, 7t, 449.

7. Rahmant. M. A. A.: Moonl. S.-S. Bull. Korean Chent. Soc. 2007. 28. 1261 . 